\title{
Convergence Rates for Nonholonomic Systems in Power Form
}

\author{
Robert T. M'Closkey* and Richard M. Murray \\ Department of Mechanical Engineering \\ California Institute of Technology \\ Pasadena, CA 91125
}

\begin{abstract}
This paper investigates the convergence rates of several controllers for low dimensional nonholonomic systems in power form. The method of multiple scales is found to be effective in determining the asymptotic form of the solutions. The general form of the perturbation solutions indicates how parameters in the control laws may be chosen to achieve a desired convergence rate. A detailed analysis of controllers exhibiting exponential convergence is included.
\end{abstract}

\section{Introduction}

This paper focuses on the problem of determining the rates of convergence of control systems of the form

$$
\dot{x}=\sum_{i=1}^{m} g_{i}(x) u_{i}(x, t) \quad x \in \mathbf{R}^{n},
$$

where each $g_{i}$ is a smooth vector field on $R^{n}$ and the controls, $u_{i}(x, t)$, are continuous functions of $x$. Systems of this form arise in the study of mechanical systems with velocity constraints and have received renewed attention as an example of strongly nonlinear systems. See [9] for an introduction and more detailed motivation. For such systems, control methods based on linearization cannot be applied and nonlinear techniques must be utilized. Convergence rates of solutions are of practical importance and we attempt to shed some light on the connection between convergence rates and the smoothness of the feedback $u_{i}(x, t)$.

A control law $u=k(x, t)$ globally stabilizes a point $x_{0} \in \mathbf{R}^{\mathbf{n}}$ if $x(t) \rightarrow x_{0}$ as $t \rightarrow \infty$ for all initial conditions of the system. For a nonholonomic control system, the dependence of a stabilizing control law on time is essential since the system (1) does not satisfy Brockett's necessary condition for smooth or even continuous stabilization [1]. Hence there does not exist a smooth static state feedback law which stabilizes the system to a point. Recent work by Coron has shown that it is possible to stabilize a nonholonomic system using time-varying feedback [2]. Constructive approaches have been presented by Samson [11], Pomet [10], and Coron and Pomet [3]. In this paper we analyze some specific nonholonomic systems in so called power form [13]. The structure of the stabilizing control laws are taken from [13]. These control laws are based on earlier work using sinusoids for open-loop planning and have connections with the recent work of Sussmann and Liu [12] and Gurvits [4].

Nonholonomic systems in power form are represented by

\footnotetext{
"Supported in part by AFOSR F49620-92J-0293

tSupported in part by a grant from the Powell Foundation
}

the following set of equations

$$
\begin{aligned}
\dot{x}_{1} & =u_{1} \\
\dot{x}_{2} & =u_{2} \\
\dot{x}_{3} & =x_{1} u_{2} \\
\dot{x}_{4} & =\frac{1}{2} x_{1}^{2} u_{2} \\
& \vdots \\
\dot{x}_{n} & =\frac{1}{(n-2) !} x_{1}^{n-2} u_{2} .
\end{aligned}
$$

The control Lie algebra for this system is spanned by the input vector fields and Lie products of the form $\mathrm{ad}_{s_{1}}^{k} g_{2}$. It is worthwhile to note that this form satisfies some of the simplifying assumptions used by Pomet to generate controllers for more general nonholonomic control systems [10].

Systems in power form characterize the fundamental difficulties of nonholonomic systems in a very simple and useful form. By understanding the geometry of controllers applied to power form, we hope to understand the geometry of controllers applied to more general nonholonomic systems. This point of view has been used very successfully by Sussmann, who has shown how results applied to a "symbolic" representation of the control system can be used to understand systems with a compatible control Lie algebra [8].

The control laws studied here are based on those presented by Teel et al. in which sinusoids at integrally related frequencies locally stabilize power form systems to a point [13]. Necessary and sufficient conditions for conversion to power form are given in this reference. Teel's controllers have the form

$$
\begin{aligned}
& u_{1}=-x_{1}-\sum_{j=1}^{n-2} x_{j+2}^{2}(\sin t-\cos t) \\
& u_{2}=-x_{2}-\sum_{j=1}^{n-2} c_{j} x_{j+2} \cos j t,
\end{aligned}
$$

with $c_{j}>0$. The proof of the stability relies on the interaction between sinusoids at integrally related frequencies to produce motion in the appropriate directions. We use essentially the same structure as (3) except that the $x_{j}$ 's are replaced by general functions of these variables. The reader is referred to [13] for more details.

We explore the convergence properties of these controls laws using multi-time scale analysis techniques and concentrating on low-dimensional cases. We are optimistic that the stabilizing controllers presented here can be extended to the more general case and that by understanding their action on a canonical system, we can understand their extension to systems with a similar Lie algebraic structure.

\section{3-Dimensional Power System}

The three dimensional nonholonomic system in power form is represented by the following system of equations:

$$
\begin{aligned}
& \dot{x_{1}}=u_{1} \\
& \dot{x_{2}}=t_{2} \\
& \dot{x_{3}}=x_{2} u_{1} .
\end{aligned}
$$


We will study the behavior of the closed loop system under the following feedback:

$$
\begin{aligned}
& u_{1}=-x_{1}+F_{1}\left(x_{3}\right) \cos t \\
& u_{2}=-x_{2}+F_{2}\left(x_{3}\right) \sin t,
\end{aligned}
$$

where $F_{1}(\cdot)$ and $F_{2}(\cdot)$ are smooth functions but otherwise unspecified. Center manifold theory may be used to choose the $F_{i}$ 's such that the origin is locally asymptotically stable in the closed loop system [13]. In order to study the rates of convergence of the solutions, it is useful to employ a two-timing perturbation procedure [7]. The method of two-timing will be used to develop the structure of some approrimate solutions of system (4) with the feedback (5). Two-timing relies upon a small system parameter which canses the flow of the system to evolve with two different time scales. Assume for the moment that the $x_{3}$ equation is replaced with

$$
\dot{x}_{3}=\epsilon x_{2} u_{1}=\epsilon x_{2}\left(-x_{1}+F_{1}\left(x_{3}\right) c_{1}\right),
$$

where $0<\epsilon<1$ and $c_{i}=\cos i t$ and $s_{i}=\sin i$. This notation will be used in the sequel. A two-timing perturbation expansion may be constructed by assuming that the solutions of the closed loop system may be written in the following form,

$$
x_{i}(t)=\bar{x}_{i}(t, \tau)+\epsilon \bar{x}_{i}(t, \tau)+\ldots
$$

where $\tau=\epsilon t$ is interpreted as slow time and $t$ as fast time. The overbar denotes the leading order terms in the expansion and the tilde denotes higher order terms. $\tau$ is treated as another independent variable in this expansion.

The difficult step in the two-timing procedure is obtaining the form of the expansion. Once the structure of the expansion is known (or assumed, as we have) it is relatively simple, although tedious, to obtain the equations for the individual terms. Substituting the expansion into the closed loop equations and equating coefficients of powers of $\epsilon$ yields the following leading order terms for $x_{1}, x_{2}$ and $x_{3}$,

$$
\begin{aligned}
\dot{\bar{x}}_{1} & =-\bar{x}_{1}+F_{1}\left(\bar{x}_{3}\right) c_{1}, \\
\dot{\bar{x}}_{2} & =-\bar{x}_{2}+F_{2}\left(\bar{x}_{3}\right) s_{1}, \\
\dot{\bar{x}}_{3} & =0, \\
\bar{x}_{3}^{\prime}+\dot{\bar{x}}_{3} & =-\bar{x}_{2}\left(\bar{x}_{1}+F_{1}\left(\bar{x}_{3}\right) c_{1}\right) .
\end{aligned}
$$

Here $\dot{x}_{i}$ denotes differentiation with respect to $t$ and $x_{i}^{\prime}$ denotes differentiation with respect to $\tau$. Equation (9) implies that $\bar{x}_{3}$ is a function of $\tau$ only,

$$
\bar{x}_{3}(t, \tau)=A_{3}(\tau) .
$$

In terms of $A_{3}(\tau)$ equations (7) and (8) may be rewritten as,

$$
\begin{aligned}
& \dot{\bar{x}}_{1}=-\bar{x}_{1}+F_{1}\left(A_{3}(\tau)\right) c_{1} \\
& \dot{\bar{x}}_{2}=-\bar{x}_{2}+F_{3}\left(A_{3}(\tau)\right) s_{1} .
\end{aligned}
$$

These equations represent first-order linear systems driven by sinusoids. Since asymptotic solutions are desired, the transient behavior of the solutions is not of interest. The steady state solutions of these equations are,

$$
\begin{aligned}
& \bar{x}_{1}(t, \tau)=\frac{1}{2} F_{1}\left(A_{3}(\tau)\right)\left(c_{1}+s_{1}\right) \\
& \bar{x}_{2}(t, \tau)=\frac{1}{2} F_{2}\left(A_{3}(\tau)\right)\left(-c_{1}+s_{1}\right) .
\end{aligned}
$$

$A_{3}(\tau)$ is determined from equation (10),

$$
A_{3}(\tau)^{\prime}+\dot{\tilde{x}}_{3}=\bar{x}_{2}\left(-\bar{x}_{1}+F_{1}\left(A_{3}\right) c_{1}\right) \text {. }
$$

Substituting the expressions in (11) into equation (10) yields,

$$
\begin{aligned}
A_{3}^{\prime}+\dot{\tilde{x}}_{3} & =-\frac{1}{4} F_{1}\left(A_{3}(\tau)\right) F_{3}\left(A_{3}(\tau)\right)\left(c_{1}-s_{1}\right)^{2} \\
& =-\frac{1}{4} F_{1}\left(A_{3}(\tau)\right) F_{2}\left(A_{3}(\tau)\right)\left(1-2 c_{1} s_{1}\right)
\end{aligned}
$$

The slow and fast time scales are considered unrelated as far as these equations are concerned. Hence $A_{3}^{\prime}$ is equated to

$$
A_{3}^{\prime}=-\frac{1}{4} F_{1}\left(A_{3}\right) F_{2}\left(A_{3}\right) \text {. }
$$

$\dot{\bar{x}}_{3}$ is equal to the remaining term

$$
\dot{\tilde{x}}_{3}=\frac{1}{2} F_{1}\left(A_{3}\right) F_{2}\left(A_{3}\right) c_{1} s_{1} .
$$

The presence of the $F_{i}(\tau)$ 's in this equation present no problems since these functions remain constant compared to the fast time, $t$. The initial conditions have been ignored so these approximate solutions will be valid after the transient beharior has died away. The magnitudes of $\bar{x}_{1}$ and $\bar{z}_{2}$, after the solution has settled down to its steady state, only depends on $\bar{x}_{3}$. It is evident from equation (12) that the rate of convergence of the $x_{3}$ variable is controlled by the product of $F_{1}$ and $F_{2}$. The following example will illustrate these ideas.

Example 1. Suppose $F_{1}$ and $F_{2}$ are chosen such that,

$$
F_{1}\left(x_{3}\right)=x_{3} \quad F_{2}\left(x_{3}\right)=x_{3}^{2},
$$

then the origin is locally asymptotically stable [13]. We introduce the parameter $\epsilon$ by scaling the dependent variables

$$
\left(x_{1}, x_{2}, x_{3}\right) \rightarrow\left(\epsilon^{\frac{1}{3}} x_{1}, \epsilon x_{2}, \epsilon^{\frac{1}{2}} x_{3}\right) .
$$

The closed loop equations transform to,

$$
\begin{aligned}
& \dot{x_{1}}=-x_{1}+x_{3} c_{1} \\
& \dot{x_{2}}=-x_{2}+x_{3}^{2} s_{1} \\
& \dot{x_{3}}=\epsilon x_{2}\left(-x_{1}+x_{3} c_{1}\right) .
\end{aligned}
$$

The equation (12) with (13) implies

$$
x_{3}(t) \sim \frac{x_{3}(0)}{\sqrt{\frac{1}{2} x_{3}^{2}(0) e t+1}} .
$$

Equations (11) yield the following asymptotic solution for $x_{1}(t)$ and $x_{3}(t)$,

$$
\begin{aligned}
& x_{1}(t) \sim \frac{1}{2} \frac{x_{3}(0)}{\sqrt{\frac{1}{2} x_{3}^{2}(0) t+1}}\left(c_{1}+s_{1}\right) \\
& x_{2}(t) \sim \frac{1}{2} \frac{x_{3}^{2}(0)}{\frac{1}{2} x_{3}{ }^{2}(0) t t+1}\left(-c_{1}+s_{1}\right) .
\end{aligned}
$$

These approximate methods usually yield estimates which are ralid only on finite time intervals. Hence, no conchusion can be made concerning the rate of convergence of the solutions without some extra analysis. Howerer, a simple calculation shows that the leading order multiscale procedure gives results equivalent to the center manifold approach when $F_{1}$ and $F_{2}$ are smooth. The perturbation solution is merely the averaged center manifold equation. Thus, the perturbation results are asymptotic in this case. Figure 1 compares the numerical solntion of system to the asymptotic results developed above. The initial conditions where chosen to minimize the transients.

\section{Control Laws for Exponential Conver- gence}

The form of the asymptotic solutions is very suggestive. It was noted earlier that the product of $F_{1}$ and $F_{2}$ controlled the convergence rate of the three state system of. equation (12).

Brample 1, continued. We now explore choices of $F_{1}$ and $F_{2}$ which give exponential reates of stabilization. The preceding analysis requires the differentiability of $F_{1}$ and $F_{2}$. Forget for 


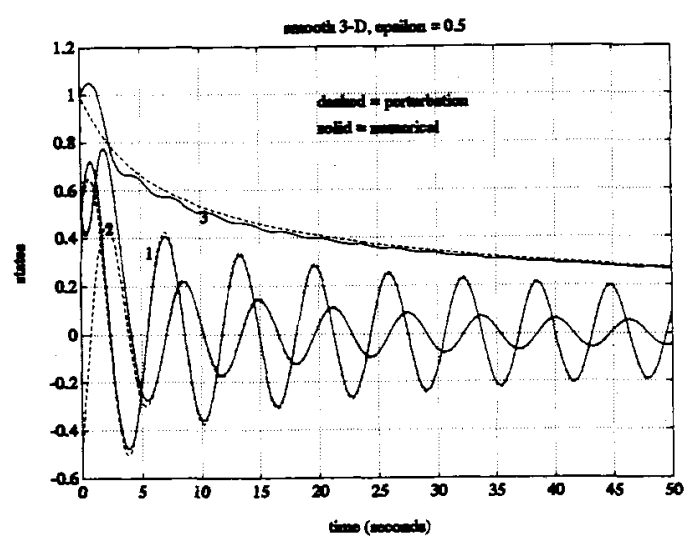

Figure 1: Comparison of numerical and perturbation solutions

the moment these restrictions and consider the 3-D closed loop system when the $F_{i}$ 's are chosen as,

$$
F_{1}\left(x_{3}\right)=\operatorname{sgn}\left(x_{3}\right) \sqrt{\left|x_{3}\right|} \quad F_{2}\left(x_{3}\right)=\sqrt{\left|x_{3}\right|},
$$

where $\operatorname{sgn}(\cdot)$ is the signum function. Once again the dependent variables may be scaled so that the closed loop equations are in the proper form,

$$
\begin{aligned}
& \dot{x}_{1}=-x_{1}+\operatorname{sgn}\left(x_{3}\right) \sqrt{\left|x_{3}\right|} c_{1} \\
& \dot{x}_{2}=-x_{2}+\sqrt{\left|x_{3}\right|} s_{1} \\
& \dot{x}_{3}=\epsilon x_{2}\left(-x_{1}+\operatorname{sgn}\left(x_{3}\right) \sqrt{\left|x_{3}\right|} c_{1}\right) .
\end{aligned}
$$

Note that the equations are Hölder continuous bet not Lipschitz at $x_{3}=0$. Continuity of the vector field ensures existence but not uniqueness of solutions. We will proceed on the assumption that the solutions are unique. Away from the set $x_{3}=0$ the rector field is Lipsehitz and the perturbation analysis indicates that for properly chosen initial conditions (where the transients are minimal) $x_{3}$ only approaches the "bad" set in the limit as $t \rightarrow \infty$.

Equation (12) implies that $\bar{x}_{3}$, and hence $\bar{x}_{1}$ and $\bar{x}_{3}$, converge exponentially. One conld argue that the asymptotic solntion is still valid with this $C^{0}$ control law since the only region in phase space where the control is not smooth is the plane $x_{3}=0$ and numerical simulations indicate that after an initial period of transient behavior the $x_{3}$ variable maintains constant sign, never passing through $x_{3}=0$. The perturbation solution is given by

$$
\bar{x}_{3}(t)=x_{3}(0) \exp \left(-\frac{\epsilon}{4} t\right)
$$

and $\bar{x}_{2}(t)$ and $\bar{x}_{2}(t)$ are given by the expressions (11). Figure 2 compares the perturbation solution with numerical integration for this nonsmooth system. Figure 3 is a semilog plot of the Euclidean norm of the states from Figure 2 and the exponential convergence rate bounds provided from the analysis. The fact the trajectories are always bounded above by a line of negative slope indicates exponential convergence. The perturbation solution pleces the exponential convergence rate bound for $x_{3}$ at $-\frac{\varepsilon}{4}$ while the $x_{1}$ and $x_{2}$ variables are half this rate. The plots show that these bounds are tight.

The availability of more rigorous theory to study nonLipschitz rector fields is required even though these hueristic arguments and simnlations are convincing. This is discussed in the following paragraphs.

The number of tools for analyzing the properties of differential equations dwindles as we pass from differentiable to

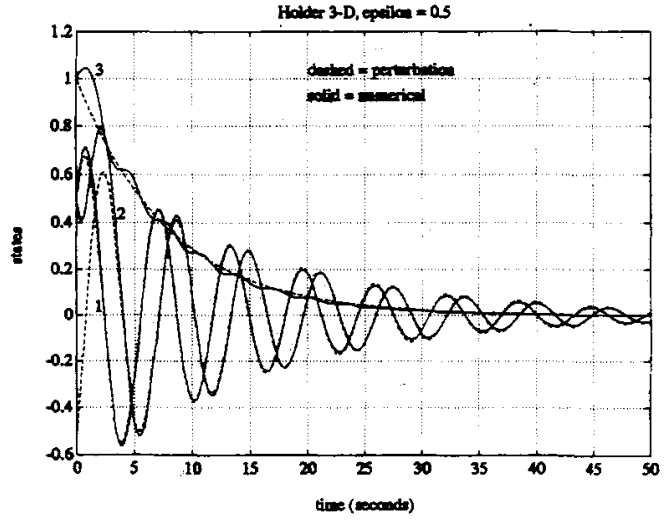

Figure 2: 3-D System with Nonsmooth Feedback

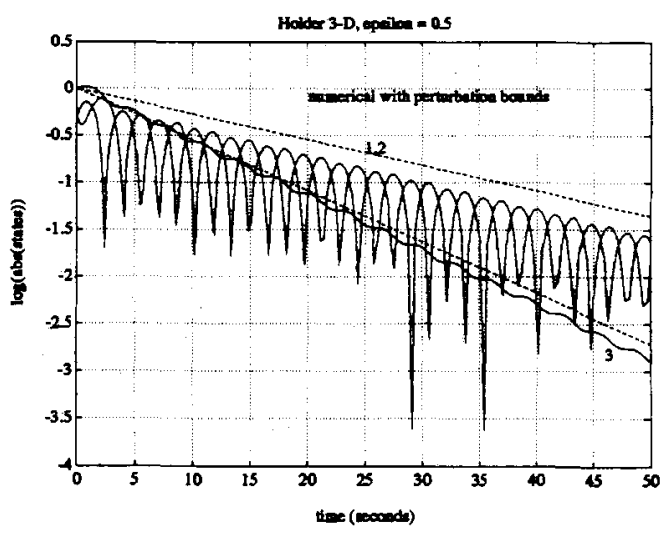

Figure 3: Exponential Rates of Convergence for 3-D System

continuous rector fields. However, Kawski has developed some nseful theory for a special class of vector fields [6]. In the interest of conserving space only those details necessary for the study of this example will be introduced.

Example 1, continued. Recall the original unscaled vector field,

$$
\begin{aligned}
& \dot{x}_{1}=-x_{1}+\operatorname{sgn}\left(x_{3}\right) \sqrt{\left|x_{3}\right|} c_{1} \\
& \dot{x}_{3}=-x_{2}+\sqrt{\left|x_{3}\right|} s_{1} \\
& \dot{x}_{3}=x_{2}\left(-x_{1}+\operatorname{sgn}\left(x_{3}\right) \sqrt{\left|x_{3}\right|} c_{1}\right) .
\end{aligned}
$$

The system of equations is invariant under the following scaling,

$$
\left(x_{1}, x_{2}, x_{3}\right) \rightarrow\left(\lambda x_{1}, \lambda x_{2}, \lambda^{2} x_{3}\right) \quad \lambda>0
$$

This scaling, called a dilation, is symbolically represented as the operator, $\delta_{\lambda} x=\left(\lambda x_{1}, \lambda x_{2}, \lambda^{2} x_{3}\right)$. Differential equations which are invariant under the dilation are termed homogeneous of order zero with respect to $\delta_{\lambda}$. It is useful to define a homogeneous norm, $\rho$, that satisfies the property $\rho\left(\delta_{\lambda} x\right)=\lambda \rho(x)$, $\lambda>0$. In this contert, a different notion of exponential stability mas be introduced [6].

Definition 1 A vector field which is homageneous of onder zero with respect to the dilation $\delta_{\lambda}$ is exponentially stable if there constants $\boldsymbol{M}>0$ and $\alpha>0$ such that,

$$
\rho(x(t)) \leq M e^{-\alpha t} \rho(x(0)),
$$

where $\rho(\cdot)$ is a corresponding homogeneous norm. 
The homogeneous norm is not equivalent to the standard $p$ norms when the variables are scaled by different factors. In this case it is impossible to substitute the usual definition of exponential stability for the one given above. Hence, based on our simulations, it is likely that the system is exponentially stable with respect to the appropriate homogeneous norm. A particular choice which satisfies the scaling property in the example is

$$
\rho(x)=\left(x_{1}^{4}+x_{2}^{4}+x_{3}^{2}\right)^{\frac{1}{4}} .
$$

This norm is useful in applications since it is smooth except at the origin. Another result by Kawski [6] states that exponential stability with respect to the homogeneous norm is equivalent to uniform asymptotic stability in the norm (just like the linear case). Since our example is time-periodic we need only show asymptotic stability. Proving asymptotic stability for a non-Lipeshitz continuous vector field is still a daunting task. However, the dilation specifies a transformation group on the phase space which facilitates the study of the equation by considering a vector field on the quotient manifold specified by the group. The quotient manifold is given by $\rho(x)=1$ which is just a warped sphere, $S^{2}$, naturally embedded in $R^{3}$. The projection map $\pi: R^{2} \backslash\{0\} \rightarrow S^{2}$ onto the sphere is

$$
\pi(x)=\left(\frac{x_{1}}{\rho(x)}, \frac{x_{2}}{\rho(x)}, \frac{x_{3}}{\rho^{2}(x)}\right) .
$$

Pushing forward the vector field with this map defines a unique vector field on the sphere by virtue of the transformation group invariance. Once the flow on the sphere is known the flow of the original vector field is determined by lifting the sphere flow with an addition scalar equation. The scalar equation is merely the differential equation for $\rho$ written in the sphere coordinates. Determining the asymptotic behavior of the full set of equations amounts to computing the nonwandering set, $\Omega$, on the sphere and observing the behavior of $\rho$ on $\Omega$. Full details for the time invariant case are found in [6]. However, we may simulate the equations on the sphere and characterize the attractive invariant sets for the time-periodic system in the extended phase space $S^{2} \times S^{1}$. This is carried out below.

The equations on the sphere $\rho(x)=1$ are,

$$
\begin{aligned}
& \dot{y}_{1}=-y_{1}+\operatorname{sgn}\left(y_{3}\right) \sqrt{\left|y_{3}\right|} c_{1}-y_{1} Q(y, t) \\
& \dot{y}_{2}=-y_{3}+\sqrt{\left|y_{3}\right| s_{1}}-y_{2} Q(y, t) \\
& \dot{y}_{3}=y_{3}\left(-y_{1}+\operatorname{sgn}\left(y_{3}\right) \sqrt{\left|y_{3}\right|} c_{1}\right)-2 y_{3} Q(y, t),
\end{aligned}
$$

where $Q(y, t)$ is given by,

$$
\begin{aligned}
Q(y, t) & =y_{1}^{3}\left(-y_{1}+\operatorname{sgn}\left(y_{3}\right) \sqrt{\left|y_{3}\right|} c_{1}\right)+y_{2}^{3}\left(-y_{2}+\sqrt{\left|y_{3}\right| s_{1}}\right) \\
& +\frac{1}{2} y_{2} y_{3}\left(-y_{1}+\operatorname{sgn}\left(y_{3}\right) \sqrt{\left|y_{3}\right|} c_{1}\right) .
\end{aligned}
$$

The scalar "radial" equation is,

$$
\dot{\rho}=Q(y, t) \rho .
$$

Simulations of the sphere equations (18) imply the existence of two stable sets on the sphere (Figure 4). The stability of the $\rho$ equation is determined by what $Q(y, t)$ does on these sets. Figure 5 shows that on the sets identified in Figure 4 that $Q(y, t)$ is periodic with a nonsero negative average. Hence $p$ is exponentially stable with the average rate of convergence equal to the average of $Q(y, t)$. This analysis is not complete until all components of $\Omega$ have been identified. Other limit sets on the sphere that aren't observable in the simulations, because they are unstable, are the points $( \pm 1,0,0)$ and $(0, \pm 1,0)$. It is obvious from the original equations that trajectories starting here converge exponentially. Hence for the six sets identified

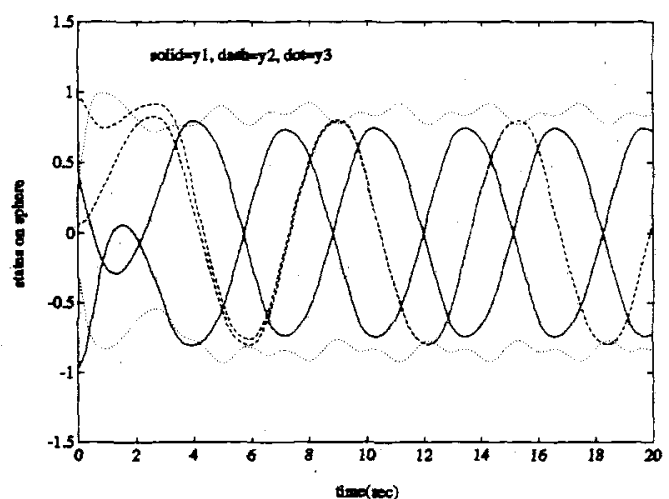

Figure 4: Stable sets on the sphere $\rho(x)=1$

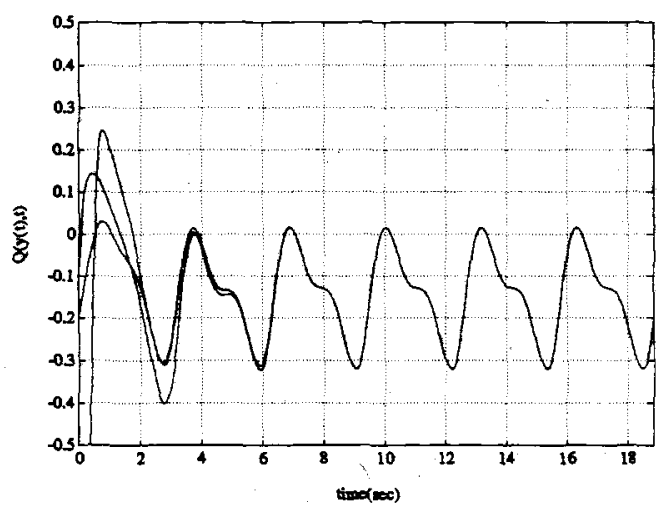

Figure 5: $\rho$ equation coefficient

on the sphere, exponential stability of the $\rho$ equation has been demonstrated.

This analysis reveals the relationship between regularity of the control law and the corresponding achievable convergence rate. We have not exhansted all of the possibilities for choosing the functional dependence of the $F_{i}$ 's for exponential stability. As long as their product is $x_{3}$ we would expeet exponential convergence. However, our choice is the most regular in terms of the Hölder continuity of the control law. Similarly, suppose a Lipschitz feedback is desired for the 3-D system. The perturbation result indicates that the fastest convergence rate possible corresponds to,

$$
\dot{\bar{x}}_{3}=-\frac{\epsilon}{4} \operatorname{sgn}\left(\bar{x}_{3}\right) \bar{x}_{3}^{2}
$$

with $F_{1}=\bar{x}_{3}$ and $F_{2}=\left|\bar{x}_{3}\right|$. A faster rate would require a continuous but not Lipschitz control.

\section{Higher Dimensional Power Systems}

Is the two-timing procedure generalizable to any system in power form? Obtaining the rates of convergence for power systems requires solving a simplified form the the closed loop equations. Since each power form system with its corresponding control law is a distinct dynamical system, one could not expect to exhibit a general algorithm for computing asymptotic solutions valid for all time. Averaging guarantees that solutions of the approximate system and original system remain close for finite time and hence does not reveal the long term behavior, even for asymptotically stable systems. Hence 
the analysis must be carried out on a case-by-case basis. We now look at the 4-dimensional power system.

Refer to the 4-dimensional nonholonomic power form system defined by the general expression of Equation 2. The structure of the control law is,

$$
\begin{aligned}
& u_{1}=-x_{1}+F_{1}\left(x_{3}\right) c_{1}+G_{1}\left(x_{4}\right) c_{2} \\
& u_{2}=-x_{2}+F_{3}\left(x_{3}\right) s_{1}+G_{2}\left(x_{4}\right) s_{1} .
\end{aligned}
$$

The $F_{i}$ 's and $G_{i}$ 's are smooth functions of their arguments. To justify a multiscale perturbation expansion we artificially introduce a parameter $\epsilon$ again. Suppose the closed loop equations are in the form,

$$
\begin{array}{ll}
\dot{x}_{1}=u_{1} & \dot{x}_{3}=\epsilon x_{2} u_{1} \\
\dot{x}_{2}=u_{2} & \dot{x}_{4}=\epsilon^{2} \frac{1}{2} x_{2}^{2} u_{1},
\end{array}
$$

with $u_{1}$ and $u_{2}$ defined above. Applying the method of multiple scales once again requires defining the form of the expansion for each variable. It is necessary to use three time scales $t$, $\tau=\epsilon t$ and $\sigma=\epsilon^{2} t$,

$$
x_{i}(t)=\bar{x}_{i}(t, \tau, \sigma)+\epsilon \bar{x}_{j}(t, \tau, \sigma)+\ldots
$$

The $\bar{x}_{i}$ 's are the leading order terms in the expansion and the $\bar{x}_{i}$ 's represent higher order terms. The computations are carried out in an analogous manner to the three state system. The leading order terms of $x_{1}$ and $x_{2}$ are merely the steady state solutions of the $\dot{x}_{1}$ and $\dot{x}_{2}$ equations when treating $x_{3}$ and $x_{4}$ as constants. These computations are suppressed to conserve space. It can be shown that $\bar{x}_{3}$ is not a function of $t$ and $\bar{x}_{4}$ is not a function of $t$ and $\tau$, hence we define,

$$
\bar{x}_{3}(t, \tau, \sigma)=A_{3}(\tau) \quad \bar{x}_{4}(t, \tau, \sigma)=A_{4}(\sigma) .
$$

$\bar{x}_{3}$ will depend on $\sigma$ but we aren't concerned with solving the higher order equations which exhibit the proper dependence since $w o$ are interested in the leading order terms only. The differential equations governing $A_{3}$ and $A_{4}$ are obtained by substituting the leading order expression for $x_{1}$ and $x_{2}$ into the $\dot{x}_{3}$ and $\dot{x}_{4}$ equations. This yields

$$
\begin{aligned}
\frac{\partial A_{3}}{\partial \tau}= & -\frac{1}{4}\left(F_{2}\left(A_{3}\right)+G_{2}\left(A_{4}\right)\right) F_{1}\left(A_{3}\right)\left(c_{1}-s_{1}\right)^{2} \\
& -\frac{1}{10}\left(F_{2}\left(A_{3}\right)+G_{2}\left(A_{4}\right)\right) G_{1}\left(A_{4}\right)\left(c_{1}-s_{1}\right)\left(4 c_{2}-2 s_{2}\right) \\
\frac{\partial A_{4}}{\partial \sigma}= & \frac{1}{16}\left(F_{2}\left(A_{3}\right)+G_{2}\left(A_{4}\right)\right)^{2}\left(c_{1}-s_{1}\right)^{2} F_{1}\left(A_{3}\right) \\
& +\frac{1}{40}\left(F_{2}\left(A_{3}\right)+G_{2}\left(A_{4}\right)\right)^{2} G_{1}\left(A_{4}\right)\left(4 c_{2}-2 s_{2}\right)
\end{aligned}
$$

The quantities which are trigonometric functions of $t$ vary rapidly compared to those which depend on $\tau$ or $\sigma$ so it is tempting to average equations (21). However, the solutions of the averaged equations do not uniformly approximate those of the original system. For example, the expression containing $G_{1}\left(\bar{x}_{4}\right)\left(c_{1}-s_{1}\right)\left(4 c_{2}-2 s_{2}\right)$ in the equation for $A_{3}$ would average to zero but this term is important for the long term behavior of $A_{3}$. It is impossible to solve equations (21) for general $F_{i}$ 's and $G_{i}$ 's so we consider a specific example again.

Example 2. Suppose we make the following choices [13],

$$
\begin{array}{ll}
F_{1}\left(\bar{x}_{3}\right)=x_{3} & G_{1}\left(\bar{x}_{4}\right)=-x_{4} \\
F_{2}\left(\bar{x}_{3}\right)=x_{3}^{2} & G_{2}\left(\bar{x}_{4}\right)=x_{4}^{2} .
\end{array}
$$

The dependent variables may be rescaled to place the closed loop equations into the form of equation (20),

$$
\left(x_{1}, x_{2}, x_{3}, x_{4}\right) \rightarrow\left(\epsilon^{\frac{1}{2}} x_{1}, \epsilon x_{2}, \epsilon^{\frac{1}{2}} x_{3}, \epsilon^{\frac{1}{2}} x_{4}\right) .
$$

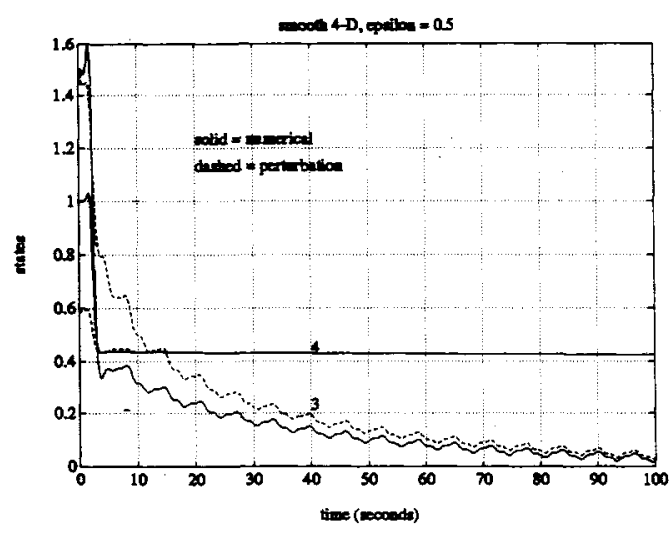

Figure 6: Smooth 4-D System, states $x_{3}$ and $x_{4}$

The differential equations for $A_{3}$ and $A_{4}$ are,

$$
\begin{aligned}
& \frac{\partial A_{3}}{\partial \tau}=-\frac{1}{4}\left(c_{1}-s_{1}\right)^{2}\left(A_{3}^{2}+A_{4}^{2}\right) A_{3} \\
& \quad-\frac{1}{10}\left(A_{3}^{2}+A_{4}^{2}\right) A_{4}\left(c_{1}-s_{1}\right)\left(-4 c_{2}+2 s_{2}\right) \\
& \frac{\partial A_{4}}{\partial \sigma}=\frac{1}{16}\left(A_{3}^{2}+A_{4}^{2}\right)^{2}\left(c_{1}-s_{1}\right)^{3} \\
& \quad+\frac{1}{40}\left(A_{3}^{2}+A_{4}^{2}\right)^{2} A_{4}\left(c_{1}-s_{1}\right)^{2}\left(-4 \cos (2 t)+2 s_{2}\right) .
\end{aligned}
$$

One could attempt an asymptotic solution of system (23). For example the averaged equations wonld yield information on the initial behavior of the solutions. The long term behavior and hence the actual rate of decay of the solutions is provided by another asymptotic solution if the analyst has enough fortitude to complete the computations. Instead it is more instructive to solve equations (23) numerically to verify that the leading order multiscale perturbation solution does in fact provide solutions that uniformly approximate the true solutions. Once this subsystem of equations has been approrimated or solved the expressions for $A_{3}$ and $A_{4}$ may be substituted back into the leading order expression of $\bar{x}_{1}$ and $\bar{x}_{2}$. Figure 6 compares the complete numerical integration of the system with the integration of the partial system of equations (23) obtained from the multiscale analysis. Only $x_{3}$ and $x_{4}$ are shown in Figure 6 since the perturbation solutions for $x_{1}$ and $x_{2}$ are just algebraic functions of $x_{3}$ and $x_{4}$.

Exponential stability of the 4-dimensional system is now considered. Analysis of the smooth 3-D example illustrated how the convergence rates of $x_{1}$ and $x_{2}$ where determined by the convergence rate of $x_{3}$. Similarly, analysis of the smooth 4 $D$ equations showed the explicit dependence of $x_{3}$ on $x_{4}$ for long time and that $x_{3}$ and $x_{4}$ essentially control the convergence of $x_{1}$ and $x_{2}$. Hence, our concern is with the subsystem formed by equations (21). Exponentially stability of the closed loop equations implies exponential stability of the averaged equations. Thus, it is reasonable to consider the averaged version of equations (21) and try to determine the $F_{i}$ 's and $G_{i}$ 's which make the averaged system exponentially stable. The averaged subsystem is,

$$
\begin{aligned}
& \frac{d A_{3}}{d \tau}=-\frac{1}{4}\left(F_{2}\left(A_{3}\right)+G_{2}\left(A_{4}\right)\right) F_{1}\left(A_{3}\right) \\
& \frac{d A_{4}}{d \sigma}=\frac{1}{40}\left(F_{2}\left(A_{3}\right)+G_{2}\left(A_{4}\right)\right)^{2} G_{1}\left(A_{4}\right) .
\end{aligned}
$$

We have abused notation by using $A_{3}$ and $A_{4}$ to represent the dependent variables in the averaged equations. To guarantee exponential stability we need to chose the highest order terms 


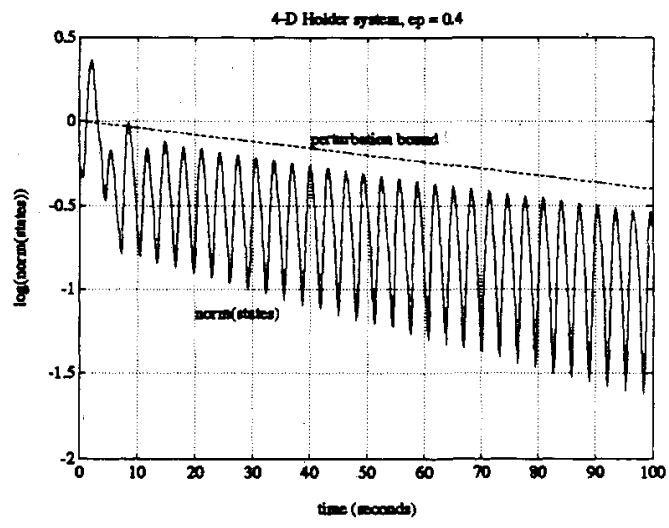

Figure 7: Exponential Rates of Convergence of 4-D System in (24) to be linear. This implies,

$$
F_{2}\left(A_{3}\right) F_{1}\left(A_{3}\right)=A_{3}, \quad G_{2}\left(A_{4}\right)^{2} G_{1}\left(A_{4}\right)=-A_{4} .
$$

One possible choice is,

$$
\begin{array}{ll}
F_{1}\left(A_{3}\right)=\operatorname{sgn}\left(A_{3}\right) \sqrt{\left|A_{3}\right|} & G_{1}\left(A_{4}\right)=-\left(A_{4}\right)^{\frac{1}{3}} \\
F_{2}\left(A_{3}\right)=\sqrt{\left|A_{3}\right|} & G_{2}\left(A_{4}\right)=\left(A_{4}\right)^{\frac{1}{t}} .
\end{array}
$$

The 4-D equations cannot be rescaled to the form (20). However, the analysis may still be valid as far as the stability type is concerned even though the relative rates of exponential convergence may not be correct. The simulations support this supposition (see Figure 7).

It is interesting to note that the $4-D$ equations with (4) are invariant under the following scaling,

$$
\left(x_{1}, x_{2}, x_{3}, x_{4}\right) \rightarrow\left(\epsilon x_{1}, \epsilon x_{2}, \epsilon^{2} x_{3}, \epsilon^{3} x_{4}\right) .
$$

This transformation is a dilation like the one described for the three dimensional example. Hence, the numerical analysis suggests that this system is exponentially stable with respect to the corresponding homogeneous norm defined by,

$$
\rho(x)=\left(x_{1}^{1} 2+x_{2}^{1} 2+x_{3}^{6}+x_{4}^{4}\right)^{\frac{1}{12}} .
$$

Computing the vector field on the sphere is carried out in an analogous manner to the 3-D case. The results are not presented. Figure 7 is a numerical simulation of the 4-dimensional nonsmooth system with the logarithm of the norm of the states plotted with respect to time. The slowest exponential convergence rate predicted by the perturbation analysis is the rate corresponding to the $x_{4}$ rariable and is given by $-\frac{c^{2}}{40}$ of. equations (24) and (4). This bound is plotted and provides a very tight estimate.

\section{Conclusions}

The analysis presented in this paper is far from complete. The 3- and 4-D systems provide some very illuminating examples to guide our thinking for higher dimensional power form and general nonholonomic control systems. Gurvitz and $\mathrm{Li}$ have made some progress in establishing a connection between the desired rate of convergence of closed loop solntions and the maximal smoothness of the fedback [5]. In the contert of exponential stabilization, their result states that the inverse of the degree of nonholonomy (the smallest order of brackets required for controllability) is an upper bound for the Hölder continuity of the control law. It is interesting to note that our control laws achieve these bounds. The 3-dimensional system has degree of nonholonomy 2 and our control laws are Hölder continuous with constant $\frac{1}{2}$. Aralogons results hold for the 4 dimensional system and our proposed exponentially stabilizing control laws.

The multi-time seale analysis is effective in providing the proper structure of the solutions. These perturbation solutions guided our choices in specifying the control parameters that determined certain convergence rates for the approximate systems.

Acknowledgements. The authors are indebted to Prof. D. Cohen for useful discussions concerning the perturbation snalysis.

\section{References}

[1] R. W. Brockett. Asymptotic stability and feedback stabilization. In R. W. Brockett, R. S. Millman, and H. J. Sussman, editors, Differential Geometric Control Theory, pages 181-191. Birkhauser, 1983.

[2] J-M. Coron. Global asymptotic stabilization for controllable systems without drift. Mathematics of Control, Signals, and Systems, 5:295-312, 1991.

[3] J-M. Coron and J-B. Pomet. A remark on the design of time-varying stabilizing feedback laws for controllable systems without drift. In IFAC Symposium on Nonlin ear Control Systems Design (NOLCOS), pages 413-417, Bordeaux, France, June 1992.

[4] L. Gurvits. A veraging approach to nonholonomic motion planning. In IEEE International Conference on Robotics and Automation, pages 2541-2546, 1992.

[5] L. Gurvits and Z.X. Li. Smooth time-periodic feedback solutions for nonholonomic motion planning. Technical memo, Robotics Research Laboratory, Courant Institute of Mathematical Sciences, 1992.

[6] M. Kawski. Homogeneous stabilizing feedback laws. Control-Theory and Advanced Technology, 6(4):497-516, 1990.

[7] J. Kevorkian and J.D. Cole. Perturbation Methods in Applied Mathematics. Springer-Verlag, 1981.

[8] G. Lafferriere and H. J. Sussmann. Motion planning for controllable systems without drift. In IEEE International Conference on Robotics and Automation pages 1148-1153, 1991.

[9] B. M. Murray and S. S. Sastry. Nonholonomic motion planning: Steering using sinusoids. IEEE Transactions on Automatic Control, May 1993. (in press).

[10] J-B. Pomet. Explicit design of time-varying stabilizing control laws for a class of controllable systems without drift. Systems and Control Letters, 18(2):147-158, 1992.

[11] C. Samson and K. Kit-Abderrahim. Feedback stabilization of a nonholonomic wheeled mobile robot. In International Conference on Intelligent Robots and Systems (IROS), 1991.

[12] H. J. Sussmann and W. Liu. Limits of highly oscillatory controls and the approximation of general paths by admissible trajectories. Technical Report SYCON-91-02, Rutgers Center for Systems and Control, 1991.

[13] A. Teel, R. M. Murray, and G. Walsh. From steering to stabilization with sinusoids. In IEEE Control and Decision Conference, pages 1603-1609, 1992. 